\title{
Aprendizagem e memória de Telenomus podisi (Hymenoptera, Platygastridae)
}

\author{
Roberta Tognon, Josué Sant'Ana \& Simone M. Jahnke
}

Departamento de Fitossanidade, Faculdade de Agronomia, Universidade Federal do Rio Grande do Sul, Av. Bento Gonçalves 7712, $91540-000$ Porto Alegre, RS, Brasil. (roberta.tognon@ufrgs. br; josue.santana@ufrgs.br; mundstock.jahnke@ufrgs.br)

\begin{abstract}
Learning and memory in Telenomus podisi (Hymenoptera, Platygastridae). The egg parasitoid Telenomus podisi Ashmead, 1893 is an important natural control agent of stink bugs. Its success as a parasitoid depends on its host-locating ability, which may be influenced by learning and memory. This study used lemongrass [Cymbopogon citratus (DC.) Stapf. (Poales, Poaceae)] extract to assess the capacity for learning and memory retention time of T. podisi at different developmental stages of the immature phase. Eggs of Euschistus heros (Fabricius, 1798) (Hemiptera, Pentatomidae) parasitized by T. podisi and at different parasitoid juvenile developmental stages were isolated in glass tubes containing filter paper with lemongrass extract or acetone solvent (control). After emergence, T. podisi females were tested in a Y-tube olfactometer containing both scents. Inexperienced females were subjected to the same scents as experienced. To assess memory, females that emerged from eggs that were in contact with lemongrass extract throughout the immature developmental stage were given a choice between lemongrass extract and acetone when they were 24, 48, 72, and 96 hours old. Females that had contact with the lemongrass during the juvenile phase preferred lemongrass, while inexperienced females or those exposed to acetone chose the solvent. The presence of lemongrass all through the immature developmental stage altered the chemotaxic orientation of T. podisi and prompted learning in these organisms that lasted for up to 72 hours.
\end{abstract}

KEYWORDS. Egg parasitoid, experience, biological control, chemotaxy.

RESUMO. O parasitoide de ovos Telenomus podisi Ashmead, 1893 é um importante agente de controle natural de percevejos e seu sucesso de parasitismo está associado à habilidade para a localização do hospedeiro, sendo a aprendizagem e a memória um dos fatores que podem influenciar esta interação. O trabalho avaliou a capacidade de aprendizagem e tempo de retenção da memória de T. podisi ao extrato de capimlimão [Cymbopogon citratus (DC.) Stapf. (Poales, Poaceae)], em diferentes estágios do desenvolvimento da fase imatura. Ovos de Euschistus heros (Fabricius, 1798) (Hemiptera, Pentatomidae) parasitados por T. podisi foram individualizados em tubos de vidro contendo papel filtro com extrato de capim-limão ou solvente acetona (controle) e mantidos em diferentes estágios do desenvolvimento jovem dos parasitoides. Após a emergência, fêmeas de T. podisi foram testadas em olfatômetro tipo "Y" aos odores de ambos tratamentos. Fêmeas inexperientes foram submetidas aos mesmos odores das experientes. Para avaliar a memória, fêmeas oriundas de ovos que permaneceram em contato com o extrato de capim-limão por todo o desenvolvimento do estágio imaturo, foram testadas, após 24, 48, 72 e 96 horas de idade ao odor de capim-limão e acetona. Fêmeas que permaneceram em contato com o capim-limão por toda a fase jovem, direcionaram-se preferencialmente para este extrato, diferente das inexperientes ou das expostas à acetona, as quais preferiram o solvente. A presença do capim-limão, durante todo o estágio do desenvolvimento imaturo, alterou a orientação quimiotáxica de T. podisi, desencadeando aprendizagem nestes organismos, a qual se manteve por até 72 horas.

PALAVRAS-CHAVE. Parasitoide de ovos, experiência, controle biológico, quimiotaxia.

A busca de parasitoides por hospedeiros inicia com um comportamento inato, no entanto, este pode ser modificado com a exposição sucessiva a determinado hospedeiro (experiência), podendo resultar em aprendizagem (VET et al., 1995; Vinson, 1998), a qual pode ser retida por diferentes períodos de tempo (memória) (Kester \& Barbosa, 1991; Cortesero et al., 1995; TAKASU \& Lewis, 1996; BJorksten \& HoffMann, 1998). A ocorrência de aprendizagem já foi observada em parasitoides braconídeos, tais como Aphidius ervi Haliday, 1834 (Du et al., 1997), Biosteres arisanus (Sonan, 1932) (DukAs \& DuAN, 2000) e Diachasmimorpha longicaudata (Ashmead, 1905) (Segura et al., 2007). A aprendizagem e o forrageamento de Trissolcus basalis (Wollaston, 1858) (Hymenoptera, Platygastridae) utilizando substâncias cairomonais de Nezara viridula L., 1758 (Hemiptera, Pentatomidae) foi estudada por DAUPHIN et al. (2009). De acordo com os autores, fêmeas experientes deste parasitoide, ou seja, que já tiveram contato com o feromônio do hospedeiro, foram mais atraídas a este odor do que as inexperientes, evidenciando que estas podem apresentar uma resposta adaptativa, ajustando o comportamento de busca a sítios de hospedeiros, de acordo com experiências do passado.

RAINs et al. (2006) constataram que o estímulo olfativo ao odor de 3-octanona, recebido na fase larval pode influenciar o comportamento quimiotáxico de adultos de Microplitis croceipes (Cresson, 1872) (Hymenoptera, Braconidae), evidenciando que a memória olfativa pode persistir após a metamorfose. Este fato já tinha sido relatado por SuJII et al. (2002), que observaram que o parasitismo de Telenomus podisi Ashmead, 1893 (Hymenoptera, Platygastridae) provenientes de ovos de Euschistus heros (Fabricius, 1798) (Hemiptera, Pentatomidae) foi maior nesta espécie do que em $N$. viridula e Acrosternun aseadum Rolston, 1983 (Hemiptera, Pentatomidae). Segundo os autores, o parasitismo é influenciado pelo hospedeiro de origem, podendo este fornecer pistas químicas que teriam influência direta na escolha e desempenho reprodutivo do parasitoide.

Telenomus podisi é um importante parasitoide de ovos de percevejos da família Pentatomidae, com potencial de uso em programas de controle biológico, pois atua como regulador populacional de diversas 
espécies, incluindo Tibraca limbativentris Stål, 1860 na cultura do arroz [Oryza sativa L. (Poales, Poaceae)] (MACIEL et al., 2007; RifFel et al., 2010) e E. heros na soja [Glycine max L. (Fabales, Fabaceae)] (MedeIros et al., 1997; PACHECo \& CorrêA-FERREIRA, 2000). Esta espécie tem sido criada em laboratório em ovos de $E$. heros, em programas de manejo e controle de percevejos da soja (PERES \& CORRÊA-FerReIRA, 2004), no entanto, pouco se sabe a respeito da influência da aprendizagem e memória no comportamento de busca de fêmeas de T. podisi. Este conhecimento é importante para que se possa, caso necessário, manipular o comportamento quimiotáxico deste parasitoide, de forma a viabilizar o uso deste organismo em programas de controle biológico de pentatomídeos. Sendo assim, este trabalho teve como objetivo avaliar a capacidade de aprendizagem e tempo de retenção da memória de T. podisi ao extrato de capimlimão [Cymbopogon citratus (DC.) Stapf. (Poales: Poaceae)], em diferentes estágios do desenvolvimento da fase imatura.

\section{MATERIAL E MÉTODOS}

A criação, manutenção e bioensaios laboratoriais foram realizados no Laboratório de Biologia, Ecologia e Controle Biológico de Insetos da UFRGS, em câmara climatizada $\left(26 \pm 1^{\circ} \mathrm{C}, 65 \pm 10 \% \mathrm{UR}\right.$, fotofase 12 horas).

Ovos de E. heros $(\mathrm{n}=20)$ com no máximo 24 horas de idade foram colados sobre uma cartela de papel $(1 \times 1,5 \mathrm{~cm})$ com fita dupla face e expostos a fêmeas de $T$. podisi, com aproximadamente 20 horas de idade, por um dia. Os lotes contendo os ovos supostamente parasitados foram individualizados em tubos de vidro e fechados com parafilme. A este recipiente foi adicionado, em diferentes períodos do desenvolvimento imaturo dos parasitoides, um papel filtro contendo $5 \mu \mathrm{L}$ de extrato de capim-limão, diluído em solvente acetona, na concentração de $2,5 \mu \mathrm{L} / \mathrm{mL}$. Após a emergência, as fêmeas foram individualizadas e testadas com no máximo 24 horas de idade, em olfatômetro tipo "Y" de vidro de dupla escolha, com diâmetro de $1,4 \mathrm{~cm}$, arena inicial de $16 \mathrm{~cm}$, bifurcada em dois "braços" de $19 \mathrm{~cm}$ cada, sob luz fluorescente (60W, luminância de 290 lux). Na extremidade de um dos braços do olfatômetro foi colocado um papel filtro de $4 \times 15 \mathrm{~cm}$, dobrado em forma de gaita, contendo $5 \mu \mathrm{L}$ do extrato de capim-limão na mesma concentração em que foram anteriormente expostos aos ovos e, na outra extremidade, adicionado um papel filtro com o mesmo volume do solvente acetona (controle). Um fluxo de ar, previamente filtrado com carvão ativo, era conduzido para dentro do sistema com o auxílio de uma bomba a vácuo conectada a um fluxímetro e um umidificador, a uma taxa de $0,8 \mathrm{~L} / \mathrm{min}$. O olfatômetro era invertido no sentido horizontal (rotação de $180^{\circ}$ ) a cada três repetições e, a cada seis, lavado com sabão neutro e hexano, sendo posteriormente seco em estufa de esterilização a $150^{\circ} \mathrm{C}$.
Após este procedimento, eram renovadas as substânciasteste. Dessa forma, realizou-se no mínimo 40 repetições para cada tratamento. As respostas foram consideradas positivas quando os parasitoides alcançavam a fonte de odor ou percorriam, pelo menos, $4 \mathrm{~cm}$ dentro dos braços e permaneciam nesta área por, no mínimo, 1 minuto. Considerou-se não responsivos os insetos que não se movimentaram nos primeiros 5 minutos ou que não alcançaram nenhum dos dois braços do olfatômetro em 10 minutos.

Os períodos de exposição dos ovos ao capimlimão foram os seguintes: (a) todo o período de desenvolvimento até a emergência (aproximadamente 12 dias); (b) apenas no início (três a quatro dias após a exposição dos ovos às fêmeas); (c) somente na fase intermediária (seis a sete dias, após esta exposição) e (d) apenas no final (nove a 11 dias, após a exposição) do desenvolvimento do parasitoide. Com exceção do primeiro tratamento, o papel filtro contendo o extrato permanecia com os ovos por 24 horas, sendo então removido do tubo. $\mathrm{O}$ mesmo procedimento descrito com capim-limão foi realizado com o solvente (acetona). Foram também conduzidos bioensaios com fêmeas inexperientes, ou seja, aquelas provenientes de ovos que não foram expostos ao capim-limão ou à acetona. Além de serem submetidas entre a escolha ao capim-limão e acetona, também foram avaliadas frente a cada um destes odores separadamente e, neste caso, contrastado com papel filtro seco, desprovido destes voláteis.

Para avaliar a memória desses insetos, fêmeas de T. podisi com no máximo 24 horas de idade, oriundas de ovos que permaneceram em contato com o extrato de capim-limão por todo o período de desenvolvimento (tratamento "a"), foram analisadas em olfatômetro tipo "Y", após 24, 48, 72 e 96 horas de idade. Estes insetos foram submetidos ao odor de capim-limão e acetona na mesma concentração e/ou volume descritos anteriormente. Nos intervalos de 24 horas entre os testes, as fêmeas permaneceram em tubos de vidro $(7,5 \times 1,3$ $\mathrm{cm}$ ), com uma gota de mel, fechado com parafilme e sem a presença dos odores. Fêmeas inexperientes, as quais nunca tiveram contato com os voláteis testados, foram submetidas ao mesmo procedimento experimental. As análises estatísticas foram realizadas com o auxílio do software BioEstat 5.0 (Ayres et al., 2007) e as diferenças nas proporções de resposta dos diferentes tratamentos foram comparadas pelo teste Qui-quadrado de heterogeneidade $(\mathrm{P}<0,05)$.

\section{RESULTADOS}

Neste estudo, a exposição de ovos parasitados de E. heros ao extrato de capim-limão durante diferentes períodos da fase jovem de $T$. podisi influenciou o comportamento quimiotáxico de fêmeas desta espécie. Os tempos de exposição com os respectivos resultados foram os seguintes: (a) quando os voláteis estiveram 
presentes durante todo o período do desenvolvimento imaturo (aproximadamente 12 dias), observou-se que $21,42 \%$ das fêmeas responderam ao controle e $66,66 \%$ ao extrato de capim-limão $\left(\chi^{2}=19,514 ; \mathrm{gl}\right.$ $=1 ; \mathrm{P}=0,0001$ ); (b) apenas no início (três a quatro dias), $52,38 \%$ direcionaram-se ao controle e $28,57 \%$ ao extrato $\left(\chi^{2}=5,882 ; \mathrm{gl}=1 ; \mathrm{P}=0,0290\right)$; (c) somente no meio do período (seis a sete dias), $40,47 \%$ foram responsivas ao controle e $50 \%$ ao extrato $\left(\chi^{2}=0,842\right.$; gl $=1 ; \mathrm{P}=0,4913)$ e (d) apenas no final (nove a 11 dias) do desenvolvimento imaturo do parasitoide, $42,85 \%$ dos insetos responderam ao controle e $47,61 \%$ ao extrato $\left(\chi^{2}\right.$ $=0,211 ; \mathrm{gl}=1 ; \mathrm{P}=0,8185$ ).

Ao serem avaliados os diferentes períodos de permanência dos parasitoides ao solvente acetona, foram observados os seguintes resultados: (a) quando o solvente esteve presente durante todo o período da fase imatura, foi verificado que $61,9 \%$ das fêmeas responderam ao solvente e $26,19 \%$ ao extrato de capimlimão $\left(\chi^{2}=12,162 ; \mathrm{gl}=1 ; \mathrm{P}=0,0011\right) ;(\mathrm{b})$ apenas no início, $64,28 \%$ direcionaram-se ao solvente e $21,42 \%$ ao extrato $\left(\chi^{2}=18 ; \mathrm{gl}=1 ; \mathrm{P}=0,0001\right)$; (c) somente no meio do período, $64,28 \%$ foram responsivas à acetona e $26,19 \%$ ao extrato $\left(\chi^{2}=13,474 ; \mathrm{gl}=1 ; \mathrm{P}=0,0006\right)$ e (d) apenas no final da fase imatura do parasitoide, $61,9 \%$ dos insetos responderam ao solvente e $28,57 \%$ ao extrato $\left(\chi^{2}=10,316 ; \mathrm{gl}=1 ; \mathrm{P}=0,0029\right)$.

Quando fêmeas inexperientes de T. podisi foram expostas, em olfatômetro, ao extrato de capim-limão e à acetona (controle), 64,28\% destas direcionaramse ao controle, diferindo significativamente das que escolheram o capim-limão $(16,66 \%)\left(\chi^{2}=23,529 ; \mathrm{gl}\right.$ $=1 ; \mathrm{P}=0,0001)$. Da mesma forma, apenas $19,04 \%$ responderam ao extrato, em comparação ao papel filtro sem odor $(73,8 \%)\left(\chi^{2}=27,128 ; \mathrm{gl}=1 ; \mathrm{P}=0,0001\right)$.
Não foi observada diferença significativa nas respostas de T. podisi ao papel filtro sem odor contrastado com o solvente acetona $\left(\chi^{2}=1,351 ; \mathrm{gl}=1 ; \mathrm{P}=0,3524\right)$.

$\mathrm{Na}$ avaliação da memória, $63,63 \%$ das fêmeas de T. podisi com 24 horas de idade, nas quais toda a fase imatura foi exposta ao tratamento capim-limão, responderam mais ao extrato em comparação às $29,54 \%$ responsivas ao controle $\left(\chi^{2}=10,976 ; \mathrm{gl}=1 ; \mathrm{P}=0,0020\right)$ (Fig. 1). Este comportamento quimiotáxico em direção ao extrato se manteve após 48 horas $\left(\chi^{2}=10,976\right.$; gl $=1 ; \mathrm{P}=0,0020)$. No entanto, passadas 72 horas de emergência, a resposta aos tratamentos não diferenciou estatisticamente $\left(\chi^{2}=0,545 ; \mathrm{gl}=1 ; \mathrm{P}=0,6225\right)$, havendo uma reversão desta aos tratamentos após 96 horas, ou seja, $58,53 \%$ responderam mais para acetona e $34,14 \%$ ao capim-limão $\left(\chi^{2}=5,263 ; \mathrm{gl}=1 ; \mathrm{P}=0,0389\right)$ (Fig. 1). Os últimos dados caracterizam o reestabelecimento do comportamento quimiotáxico inato desta espécie, ou seja, a preferência ao odor de acetona em detrimento ao capim-limão.

Fêmeas de T. podisi inexperientes, as quais na fase jovem não foram expostas aos tratamentos descritos anteriormente, foram mais responsivas à acetona (controle), independentemente da idade em que foram testadas $(\mathrm{P}<0,05)$ (Fig. 2).

\section{DISCUSSÃO}

A ausência de atratividade aos voláteis de capim-limão de fêmeas inexperientes era uma resposta esperada, tendo em vista que estes, em sua composição, possuem o citral, substância repelente a insetos, como Aedes aegypti (L., 1762) (Diptera, Culicidae) (TAwaTsIN et al., 2001).

Quanto à exposição da acetona, os resultados

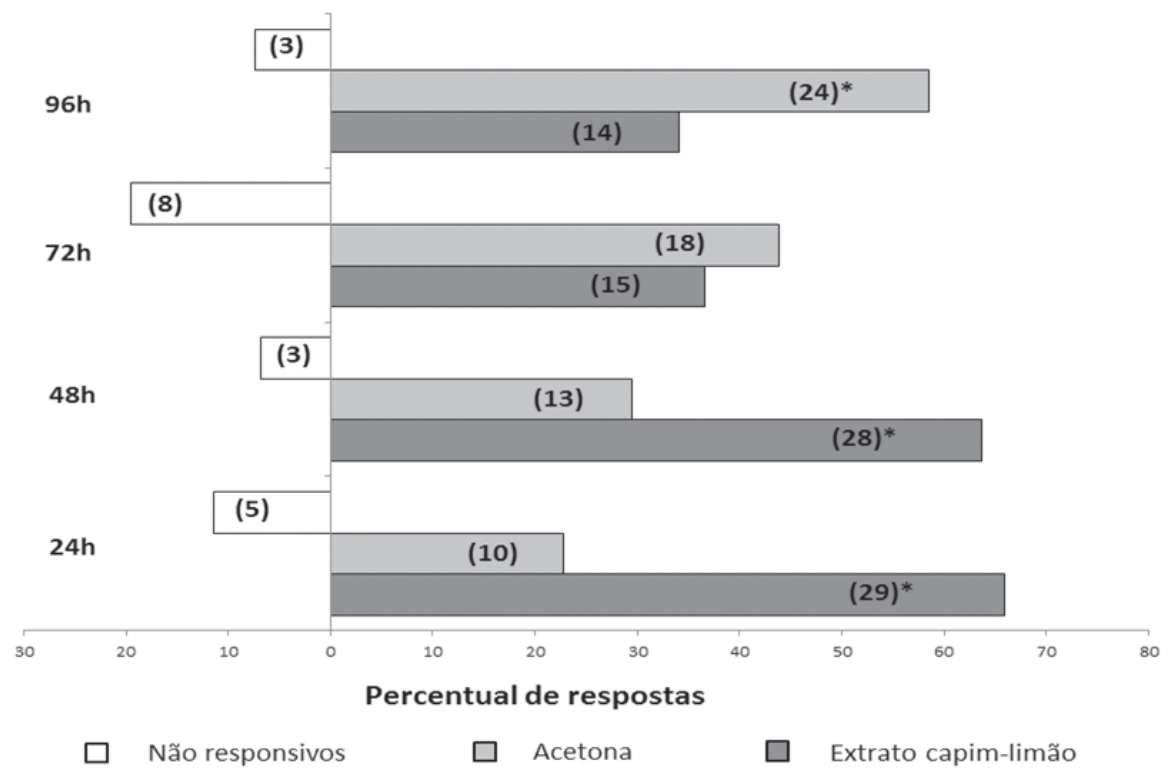

Fig. 1. Percentuais de respostas quimiotáxicas de fêmeas de Telenomus podisi tratadas com extrato de capim-limão por todo o período do desenvolvimento imaturo, testadas em olfatômetro de dupla escolha e submetidas aos voláteis de capim-limão e acetona (controle). Números entre parênteses representam a quantidade de insetos responsivos, ou não, aos tratamentos. Valores seguidos de asterisco diferem significativamente $\left(\chi^{2}, \mathrm{P}<0,05\right)$ do controle ou do extrato em cada hora. 


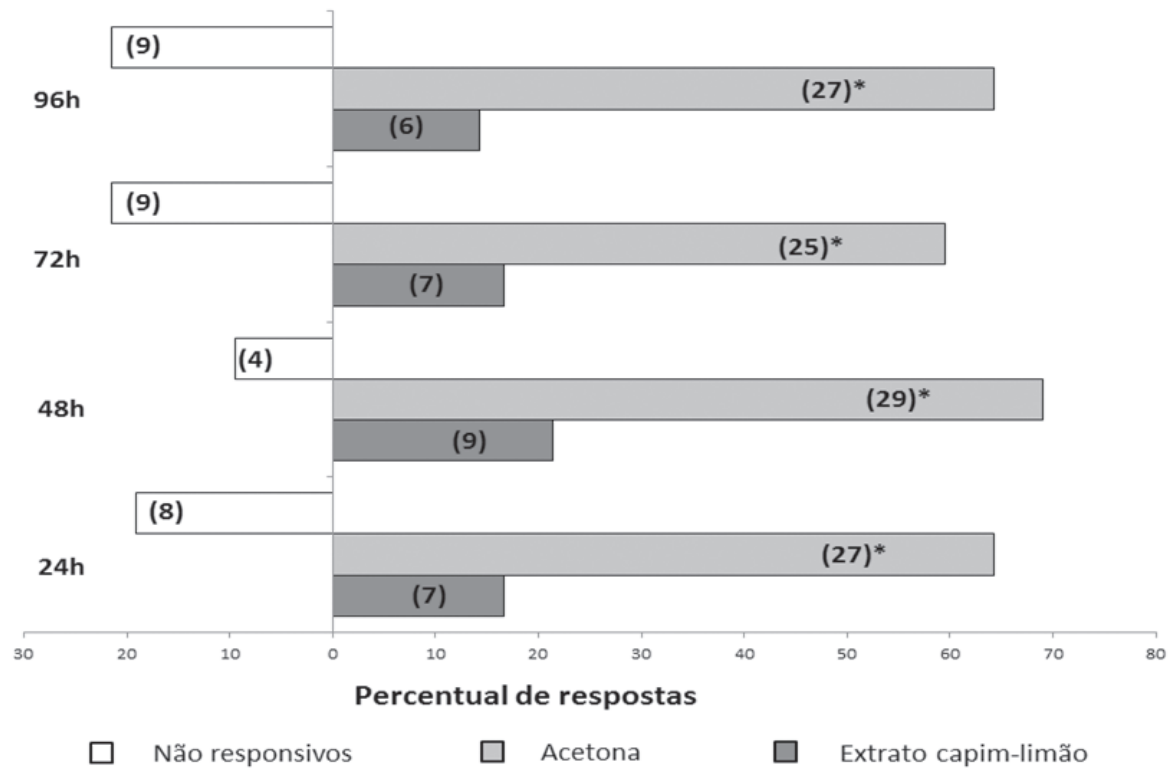

Fig. 2. Percentuais de respostas quimiotáxicas de fêmeas inatas de Telenomus podisi testadas em olfatômetro de dupla escolha e submetidas aos voláteis de capim-limão e acetona (controle). Números entre parênteses representam a quantidade de insetos responsivos, ou não, aos tratamentos. Valores seguidos de asterisco diferem significativamente $\left(\chi^{2}, \mathrm{P}<0,05\right)$ do controle em cada hora.

apontamqueindependentementedoperíodo de exposição, fêmeas de $T$. podisi mantém o comportamento inato, observado nas inexperientes. No entanto, a presença do capim-limão durante todo ou a partir da metade do período de desenvolvimento embrionário do parasitoide, altera sua orientação quimiotáxica, favorecendo o processo de aprendizagem nestes organismos. Desta forma, o parasitoide provavelmente passou a relacionar o odor, mesmo sendo originalmente repelente ao inseto, à presença da planta e/ou do hospedeiro relacionado a esta.

A relação entre o odor com o qual o parasitoide esteve em contato durante o estágio imaturo e seu hospedeiro foi relatada por BJORKSTEN \& HoFFMANN (1998), comprovando a aprendizagem em Trichogramma aff. brassicae Bezdenko, 1968 (Hymenoptera, Trichogrammatidae), pois fêmeas que se desenvolveram e emergiram de ovos de Sitotroga cerealella Oliver, 1789 (Lepidoptera, Gelechiidae) depositados sobre folhas de tomateiro, buscaram pela planta que apresentava o mesmo odor após a emergência. De forma contrária, as fêmeas que receberam o estímulo da planta somente após a emergência não apresentaram comportamento de busca por este odor. Resultado semelhante foi obtido por DukAS \& DUAN (2000), os quais observaram que o parasitoide de ovos $B$. arisanus associa os voláteis de folhas ou de frutas de goiabeira com a presença do hospedeiro.

Nas interações tritróficas (planta-herbívoroinimigo natural), uma substância liberada pela planta pode atuar como sinomônio, atraindo inimigos naturais do herbívoro que a está atacando (VINSON, 1984). Sendo assim, os sinomônios são de grande importância não só para a planta que os utiliza como um mecanismo de defesa contra o ataque do herbívoro, mas também para os inimigos naturais, que fazem uso destas pistas químicas para o encontro de hospedeiros (CONTI \& Colazza, 2012). Dessa forma, é possível que $T$. podisi tenha aprendido a relacionar o odor de capim-limão a um sinomônio, em estratégias de localização de ovos.

A mudança de comportamento de fêmeas aos voláteis do capim-limão ocorreu quando os ovos parasitados foram expostos por 24 horas ao odor na fase intermediária (tratamento "c") e final (tratamento "d") ou quando toda a fase imatura esteve na presença do volátil, resultando em aprendizado o qual se manteve por até 72 horas, caracterizando a memória. Resultado semelhante foi observado em Hyssopus pallidus (Askew, 1964) (Hymenoptera, Eulophidae), um parasitoide de larvas de Cydia pomonella L., 1758 (Lepidoptera, Tortricidae) (Matthews \& Matthews, 2010). De acordo com os autores, adultos que não haviam sido expostos ao extrato de maçã na fase imatura não apresentaram resposta significativa a este odor, mas quando permaneceram em contato com o extrato durante todo o período larval, responderam positivamente ao tratamento.

A persistência da aprendizagem, ou seja, a retenção da memória em insetos parasitoides, foi descrita para várias espécies. PERI et al. (2006) constataram que a memória de $T$. basalis foi mantida por até 23 horas após o primeiro contato deste inseto com pistas químicas deixadas no substrato como pegadas/footprint de N. viridula. Fêmeas de Eupelmus vuilleti Crawford, 1913 (Hymenoptera, Eupelmidae) mantiveram a memória por seis e sete dias quando expostas aos voláteis de Bruchidius atrolineatus (Pic, 1932) (Coleoptera, Bruchidae) e aos odores de sementes hospedeiras do inseto herbívoro, respectivamente (CORTESERo et al., 1995). Resultado semelhante foi verificado em fêmeas de Cotesia congregata (Say, 
1836) (Hymenoptera, Braconidae), que mantiveram a memória por até sete dias ao odor de cerejeira silvestre, a qual estava associada ao seu hospedeiro Manduca sexta (L., 1763) (Lepidoptera, Sphingidae) (KeSTER \& BARbosa, 1991). Para M. croceipes, a aprendizagem a três odores específicos (extratos de baunilha, de laranja e cariofileno), oferecidos juntamente com a alimentação mas isolados entre si, durou aproximadamente cinco dias (TAKASU \& LEWIS, 1996).

A aquisição da aprendizagem pode variar de acordo com o estágio de desenvolvimento larval o qual está relacionado à formação de lóbulos cerebrais. Segundo BARRon \& Corbet (1999), durante o processo de desenvolvimento os insetos holometábolos passam por uma série de transformações as quais também resultam em alterações no sistema nervoso. LeE et al. (1999) rastrearam, através da metamorfose, os neurônios do corpo cogumelar (CC) do sistema nervoso de Drosophila sp. (Diptera, Drosophilidae). Segundo os autores, o desenvolvimento dos três lóbulos do CC ocorre de maneira sequencial: o lóbulo $\gamma$ é formado na fase larval, o lóbulo $\alpha^{\prime} / \beta$ ' se desenvolve apenas antes da pupação, na metade do terceiro instar, e a neurogênese do $\alpha / \beta$ começa no início da formação da pupa. Durante esta fase os neurônios do lóbulo $\gamma$ sofrem histólise, desaparecendo, enquanto os neurônios $\alpha^{\prime} / \beta^{\prime}$ e $\alpha / \beta$ mantém suas projeções intactas após a metamorfose.

Se em T. podisi o processo de desenvolvimento for análogo ao de Drosophila sp., tanto os ovos expostos aos odores durante todo o período imaturo, quanto os expostos ao odor posteriormente à metade deste, sofreram o processo de condicionamento após a formação do lóbulo $\alpha^{\prime} / \beta^{\prime}$, o qual se mantém intacto através do processo de metamorfose.

Os resultados obtidos fornecem indícios de que é possível fazer com que $T$. podisi aprenda a reconhecer e retenha a memória de voláteis durante a fase imatura. Neste caso, posturas parasitadas, independentemente do hospedeiro de origem, poderiam ser expostas a odores de ovos ou mesmo ao feromônio de hospedeiros aos quais se destinaria ao controle, possibilitando aprendizagem e maximizando, desta forma, o comportamento de busca de espécie(s) alvo e, possivelmente, o sucesso de parasitismo. O conhecimento da interferência dos fatores que intermediam a comunicação hospedeiro-parasitoide poderá auxiliar em programas de controle biológico, possibilitando maior adequação e confiabilidade na utilização desta ferramenta com o uso de T. podisi.

\section{REFERÊNCIAS BIBLIOGRÁFICAS}

Ayres, M.; Ayres Jr, M.; Ayres, D. L. \& Santos, A. A. S. 2007. BioEstat 5.0 Aplicações estatísticas nas áreas da ciências biomédicas. Belém, Sociedade Civil Mamirauá. 339p.

Barron, A. B. \& Corbet, S. A. 1999. Preimaginal conditioning in Drosophila revisited. Animal Behaviour 58(1):621-628.

Bjorksten, T. A. \& HoffmanN, A. A. 1998. Plant cues influencing searching behaviour and parasitism in the egg parasitoid Trichogramma nr. brassicae. Ecological Entomology 23(4):355-362.
Conti, E. \& Colazza, S. 2012. Chemical ecology of egg parasitoids associated with true bugs. Psyche 2012(1):1-11.

Cortesero, A. M.; Monge, J. P. \& Huignard, J. 1995. Influence of two successive learning processes on the response of Eupelmus vuilleti Crw (Hymenoptera: Eupelmidae) to volatile stimuli from hosts and host plants. Journal of Insect Behavior 8(6):751-762.

Dauphin, G.; Coquillard, P.; Colazza, S.; Peri, E. \& Wajnberg, E. 2009. Host kairomone learning and foraging success in an egg parasitoid: a simulation model. Ecological Entomology 34(2):193-203

Du, Y.; Poppy, G. M. \& Wadhams, L. J. 1997. Chemically mediated associative learning in the host foraging behavior of the aphid parasitoid Aphidius ervi (Hymenoptera: Braconidae). Journal of Insect Behavior 10(4):509-522.

DukAs, R. \& DuAn, J. J. 2000. Potential fitness consequences of associative learning in a parasitoid wasp. Behavioral Ecology 11(5):536-543

Kester, K. M. \& Barbosa, P. 1991. Post-emergence learning in the insect parasitoid, Cotesia congregata (Say) (Hymenoptera: Braconidae). Journal of Insect Behavior 4(6):727-742.

Lee, T.; Lee, A. \& Luo, L. Q. 1999. Development of the Drosophila mushroom bodies: sequential generation of three distinct types of neurons from a neuroblast. Development 126:4065-4076.

Maciel, A. A. S.; Lemos, R. N. S.; Souza, J. R.; Costa, V. A.; Barrigossi, J. A. F. \& Chagas, E. F. 2007. Parasitismo de ovos de Tibraca limbativentris Stal (Hemiptera: Pentatomidae) na cultura do arroz no Maranhão. Neotropical Entomology 36(4):616-618.

Matthews, R. W. \& Matthews, J. R. 2010. Insect Behavior. New York, John Wiley \& Sons. 514p.

Medeiros, M. A.; Schimidt, F. V. G.; Loiácono, M. S.; Carvalho, V. F. \& Borges, M. 1997. Parasitismo e predação em ovos de Euschistus heros (Fab.) (Heteroptera: Pentatomidae) no Distrito Federal, Brasil. Anais da Sociedade Entomológica do Brasil 26(2):397-401

Pacheco, D. J. P. \& CorrêA-Ferreira, B. S. 2000. Parasitismo de Telenomus podisi Ashmead (Hymenoptera: Scelionidae) em Populações de Percevejos Pragas da Soja. Anais da Sociedade Entomológica do Brasil 29(2):295-302.

Peres, W. A. A. \& CorrêA-Ferreira, B. S. 2004. Methodology of mass multiplication of Telenomus podisi Ash. and Trissolcus basalis (Woll.) (Hymenoptera: Scelionidae) on eggs of Euschistus heros (Fab.) (Hemiptera: Pentatomidae). Neotropical Entomology 33(4):457-462.

Peri, E.; Sole, M. A.; Wajnberg, E. \& Colazza, S. 2006. Effect of host kairomones and oviposition experience on the arrestment behavior of an egg parasitoid. Journal of Experimental Biology 209(18):3629-3635.

RaIns, G. C.; UtLey, S. L. \& Lewis, W. J. 2006. Behavioral monitoring of trained insects for chemical detection. Biotechnology Progress 22(1):2-8.

Riffel, C. T.; Prando, H. F. \& Boff, M. I .C. 2010. Primeiro relato de ocorrência de Telenomus podisi (Ashmead) e Trissolcus urichi (Crawford) (Hymenoptera: Scelionidae) como parasitóides de ovos do percevejo-do-colmo-do-arroz, Tibraca limbativentris (Stål) (Hemiptera: Pentatomidae), em Santa Catarina. Neotropical Entomology 39(3):447-448. Segura, D. F.; Viscarret, M. M.; Paladino, L. Z. C.; Ovruski, S. M. \& Cladera, J. L. 2007. Role of visual information and learning in habitat selection by a generalist parasitoid foraging for concealed hosts. Animal Behaviour 74(1):131-142

Suji, E. R.; Costa, M. L. M.; Pires, C. S. S.; Colazza, S. \& Borges, M. 2002. Inter and intra-guild interactions in egg parasitoid species of the soybean stink bug complex. Pesquisa Agropecuária Brasileira 37(11):1541-1549.

TAKASU, K. \& LEwIS, W. J. 1996. The role of learning in adult food location by the larval parasitoid, Microplitis croceipes (Hymenoptera: Braconidae). Journal of Insect Behavior 9(2):265-281.

Tawatsin, A.; Wratten, S. D.; Scott, R. R.; Thavara, U. \& Techadamrongsin, Y. 2001. Repellency of volatile oils from plants against three mosquito vectors. Journal of Vector Ecology 26(1):76-82.

Vet, L. E. M.; Lewis, W. J. \& CARdÉ, R. T. 1995. Parasitoid foraging and learning. In: CARDÉ, R. T. \& BELL, W. J. Chemical ecology of insects 2. New York, Chapman \& Hall. v. 2, p. 65-101. 
VINSON, S. B. 1984. Parasitoid-host relationships. In: CARDÉ, R. T. \& Bell, W. J. eds. Chemical Ecology of Insects. New York, Chapman \& Hall. v. 1, p. 205-233.
Vinson, S. B. 1998. The general host selection behavior of parasitoid Hymenoptera and a comparison of initial strategies utilized by larvaphagous and oophagous species. Biological Control 11(2):79-96. 